\title{
Proportion of Family Physicians in Solo and Small Practices is on the Decline
}

\author{
Anuradha Jetty, MPH, Stephen Petterson, PhD, and Yalda Jabbarpour, MD
}

Although solo and small practices are a vital part of primary care, the proportion of family physicians reporting working in practices with 5 or fewer providers declined from $15 \%$ to $11 \%$ for solo and $37 \%$ to $34 \%$ for small ( 2 to 5 providers) practices from 2014 to 2018 . These decreasing trends are concerning, mainly when a low proportion of family physicians have solo practices in rural locations given the access to care challenges in these underserved populations. ( J Am Board Fam Med 2021;34:266-267.)

Keywords: Access to Health Care, Child Health, Epidemiologic Studies, Family Medicine, Family Physicians, Primary Health Care, Private Practice, Vulnerable Populations

Small and solo practices have been the traditional organizational structure for care delivery in the United States, but widescale consolidation is threatening their viability. Studies have demonstrated that small practice size is associated with fewer preventable patient hospitalizations and better access to care for patients. ${ }^{1}$ On the other hand, a larger practice size may allow for more leveraging power with payers, more capital for infrastructure improvements, such as electronic health records, and greater access to shared resources such as imaging centers or specialists. ${ }^{2,3}$ A growing number of recent family medicine graduates prefer to work in large practices for a better work-life balance and to avoid the administrative burdens and other responsibilities associated with owning a practice. ${ }^{3,4}$ It is unclear if these advantages lead to fewer family physicians (FPs) in small and solo practices.

We used the 2014 to 2018 American Board of Family Medicine (ABFM) Family Medicine Certification Examination Application data on practice size

This article was externally peer reviewed.

Submitted 31 August 2020; revised 30 October 2020; accepted 4 November 2020.

From The Robert Graham Center, Washington, DC (AJ, $\mathrm{SP}, \mathrm{YJ})$.

Funding: None.

Conflict of interest: nothing to disclose.

Author contributions: All authors made a substantial contribution to the research and in writing the policy brief, and are responsible for its content jointly and individually.

Corresponding author: Anuradha Jetty, MPH, Robert Graham Center, 1133 Connecticut Ave, NW \#1100, Washington, DC 20036 (E-mail: ajetty@aafp.org). of respondents' primary practice. We calculated the proportion of FPs seeking to continue their certification reporting solo, small (2 to 5 physicians and clinicians), medium (6 to 20$)$, and large practice size $(\geq 20)$ for the entire 2014 to 2018 sample and stratified by the rural-urban location of their primary practice. We used the Cochrane-Armitage test to determine significant differences in trends in proportions across years in each of the practice size categories.

A total of 35625 FPs providing direct patient and continuity care (we excluded FPs who reported emergency department/hospital as their primary practice site) were included in the analysis sample. (Figure 1). The number of FPs per year in the sample ranged from 5525 to 8216 . We observed a steady decline in the proportions of solo $(14 \%$ to $11 \%, P<.01)$ and small (36\% to $32 \%, P<.01)$ size practices in urban areas. In rural areas, there was a decrease of 5 percentage-points in FPs reporting solo practices, but the share of FPs in small practices remained nearly the same over time.

We find that FPs practicing in solo and small practices decreased over time in urban and solo practices in rural areas. These results are consistent with a previous study that demonstrated a $31 \%$ relative decrease in FPs in solo practices from 1992 to $2013(16 \%$ to $11 \%) .^{5}$ These findings may reflect the ongoing trends in consolidation, which disproportionately affects rural practices that may have fewer resources to sustain market pressure. The current Coronavirus disease-19 (COVID-19) pandemic could exacerbate the decline in solo and 
Figure 1. Trends in percentage of family physicians recertifying with The American Board of Family Medicine who report size of their primary practice (2014 to 2018). Source: American Board of Family Medicine

Certification Examination Application Survey $(2014$ to 2018, $N=35$ 625; 2014, $n=8216 ; 2015, n=8386 ; 2016$, $\mathrm{n}=7137 ; 2017, \mathrm{n}=6361 ; 2018, \mathrm{n}=5525)$. Analytical sample restricted to family physicians in direct patient care and providing continuity of care (Large, 20 or more providers; Medium, 6-20 providers; Small, $2-5$ providers).

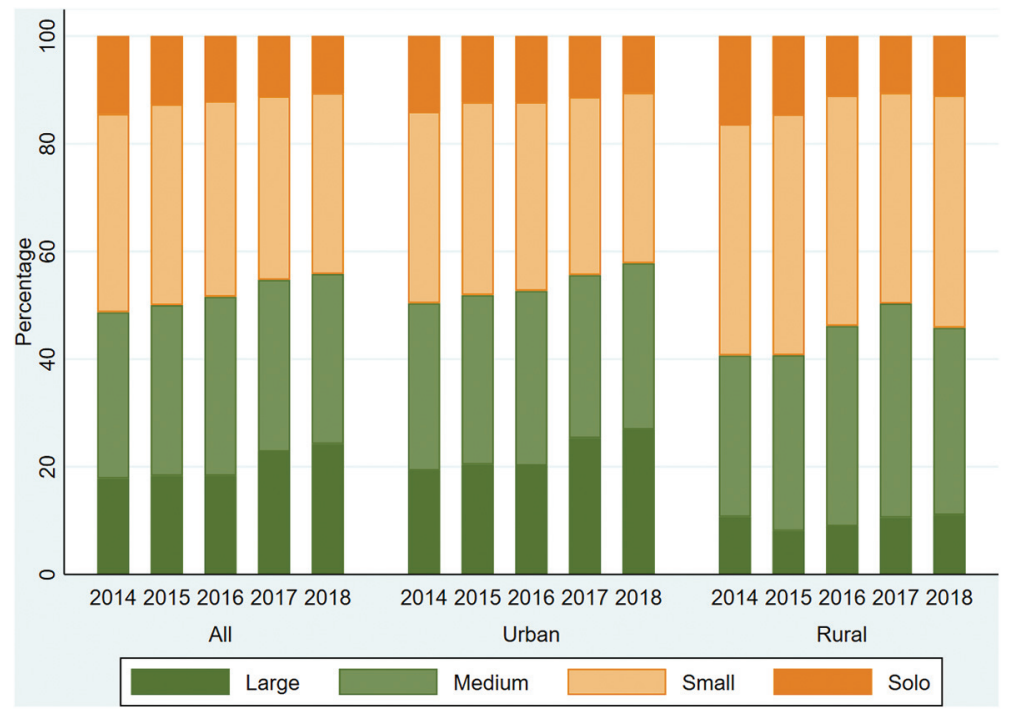

small practices further, putting many practices at risk of closure. As this study is based on survey data, there may be reporting bias the practice size may not reflect the true structure of the practice. For instance, family physicians in large practices may have either reported the local clinic unit size as practice size or the large primary practice size.

Whether this trend will have an impact on the quality of care overall is debatable, since previous studies have been mixed on practice size and quality of care. ${ }^{6,7}$ But understanding the mechanisms behind the loss of small and solo practices, whether it be by consolidation or physician choice, will likely help maintain the diversity of organizational structures in primary care.

To see this article online, please go to: http://jabfm.org/content/ 34/2/266.full.

\section{References}

1. Casalino LP, Pesko MF, Ryan AM, et al. Small primary care physician practices have low rates of pre- ventable hospital admissions. Health Aff (Millwood) 2014;33:1680-8.

2. Casalino LP, Pham H, Bazzoli G. Growth of singlespecialty medical groups. Health Aff (Millwood) 2004;23:82-90.

3. Muhlestein DB, Smith NJ. Physician consolidation: rapid movement from small to large group practices, 2013-15. Health Aff (Millwood) 2016;35:1638-42. Sep 1.

4. Eden AR, Barreto T, Hansen ER. Experiences of new family physicians finding jobs with obstetrical care in the USA. Fam Med Community Health 2019;7:e000063.

5. Peterson LE, Baxley E, Jaén CR, Phillips RL. Fewer family physicians are in solo practices. J Am Board Fam Med 2015 Jan 1;28:11-2.

6. Rittenhouse DR, Casalino LP, Shortell SM, et al. Small and medium-size physician practices use few patient-centered medical home processes. Health Aff (Millwood) 2011;30:1575-84.

7. $\mathrm{Ng} \mathrm{CW}, \mathrm{Ng} \mathrm{KP}$. Does practice size matter? Review of effects on quality of care in primary care. Br J Gen Pract 2013;63:e604-e610. 